\title{
Electromagnetic field enhancement effects in group IV semiconductor nanowires. A Raman spectroscopy approach
}

J. L. Pura, J. Anaya, J. Souto, A. C. Prieto, A. Rodríguez, T. Rodríguez, P. Periwal, T. Baron, and J. Jiménez "This article may be downloaded for personal use only.

Any other use requires prior permission of the author and AIP Publishing. This article appeared in:

Citation: Journal of Applied Physics 123, 114302 (2018); doi: 10.1063/1.5012987

View online: https://doi.org/10.1063/1.5012987

View Table of Contents: http://aip.scitation.org/toc/jap/123/11

Published by the American Institute of Physics

\section{Articles you may be interested in}

Room-temperature 2- $\mu \mathrm{m}$ GeSn P-I-N homojunction light-emitting diode for inplane coupling to group-IV waveguides

Applied Physics Letters 111, 141105 (2017); 10.1063/1.4999395

Sub-monolayer quantum dot quantum cascade mid-infrared photodetector

Applied Physics Letters 111, 251104 (2017); 10.1063/1.5011239

Optical absorption edge broadening in thick InGaN layers: Random alloy atomic disorder and growth mode induced fluctuations

Applied Physics Letters 112, 032106 (2018); 10.1063/1.5010879

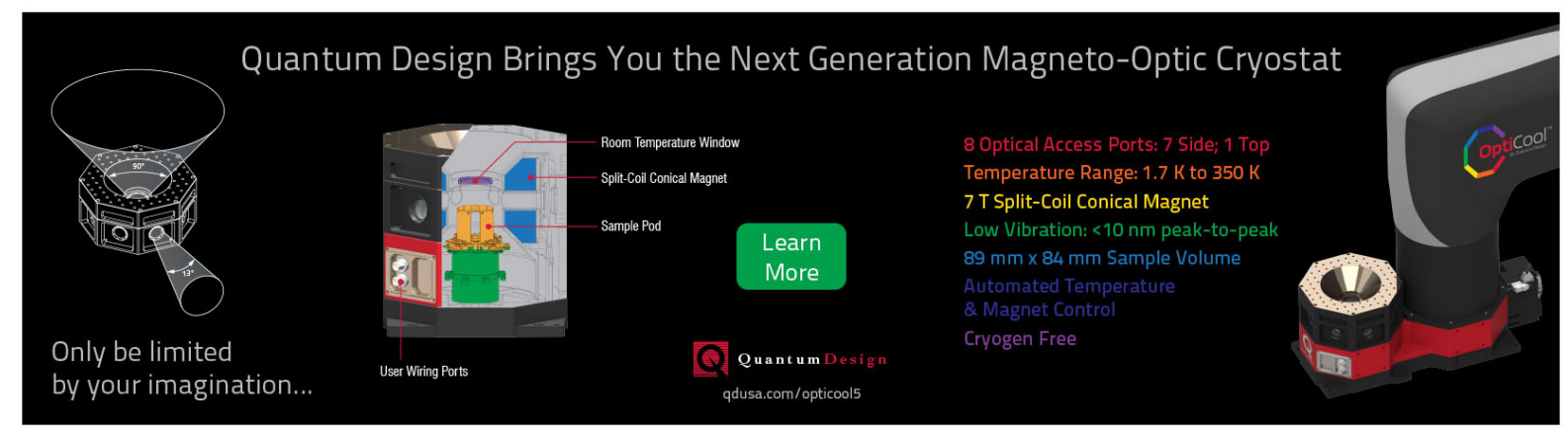




\title{
Electromagnetic field enhancement effects in group IV semiconductor nanowires. A Raman spectroscopy approach
}

\author{
J. L. Pura, ${ }^{1}$ J. Anaya, ${ }^{2}$ J. Souto, ${ }^{1}$ A. C. Prieto, ${ }^{1}$ A. Rodríguez, ${ }^{3}$ T. Rodríguez, ${ }^{3}$ P. Periwal, ${ }^{4}$ \\ T. Baron, ${ }^{4}$ and J. Jiménez ${ }^{1, a)}$ \\ ${ }^{1}$ GdS Optronlab, Dpt. Física de la Materia Condensada, ed. LUCIA Universidad de Valladolid, \\ Paseo de Belén 11, 47011 Valladolid, Spain \\ ${ }^{2} \mathrm{HH}$ Wills Physics Laboratory, University of Bristol, Tyndall Avenue, BS8 ITL Bristol, United Kingdom \\ ${ }^{3}$ Ingeniería Electrónica, ETSIT, Universidad Politécnica de Madrid, 28040 Madrid, Spain \\ ${ }^{4}$ University Grenoble Alpes, LTM, F-38000 Grenoble, France and CNRS, LTM, F-38000 Grenoble, France
}

(Received 9 November 2017; accepted 27 February 2018; published online 16 March 2018)

\begin{abstract}
Semiconductor nanowires (NWs) are the building blocks of future nanoelectronic devices. Furthermore, their large refractive index and reduced dimension make them suitable for nanophotonics. The study of the interaction between nanowires and visible light reveals resonances that promise light absorption/scattering engineering for photonic applications. Micro-Raman spectroscopy has been used as a characterization tool for semiconductor nanowires. The light/nanowire interaction can be experimentally assessed through the micro-Raman spectra of individual nanowires. As compared to both metallic and dielectric nanowires, semiconductor nanowires add additional tools for photon engineering. In particular, one can grow heterostructured nanowires, both axial and radial, and also one could modulate the doping level and the surface condition among other factors than can affect the light/NW interaction. We present herein a study of the optical response of group IV semiconductor nanowires to visible photons. The study is experimentally carried out through micro-Raman spectroscopy of different group IV nanowires, both homogeneous and axially heterostructured $(\mathrm{SiGe} / \mathrm{Si})$. The results are analyzed in terms of the electromagnetic modelling of the light/nanowire interaction using finite element methods. The presence of axial heterostructures is shown to produce electromagnetic resonances promising new photon engineering capabilities of semiconductor nanowires. Published by AIP Publishing.
\end{abstract}

https://doi.org/10.1063/1.5012987

\section{INTRODUCTION}

Semiconductor nanowires (NWs) are one-dimensional structures allowing the engineering of photons, phonons, electrons, and plasmons, which grants them a paramount role in nanodevice development. Significant differences with the behaviour of bulk materials are enabled by the large aspect ratio and the size of the confined dimension, the NW diameter. ${ }^{1-4}$ In particular, the interaction of semiconductor NWs with light presents very promising features for advanced photonic devices, e.g., photovoltaic cells, photodetectors, and light emitters, including lasers. In this frame, a broad spectrum of amazing optical properties has been reported for deep subwavelength diameter semiconductor NWs, e.g., waveguiding, ${ }^{4}$ optical resonances, ${ }^{5}$ and antenna effects. ${ }^{6}$ It has been shown that light couples to NWs in different ways depending on the NW diameter, NW composition, light wavelength, and the dielectric mismatch between the NW and the surrounding media. ${ }^{7}$ All these variables allow tuning the optical properties of the NWs. One of the most relevant properties concerning the light/NW interaction is the ability of NWs to enhance the optical absorption/scattering for certain NW diameters. ${ }^{4-6,8-11}$ Different phenomena related to such a resonance effect have been reported, e.g., enhanced photocurrent in Ge NWs, ${ }^{8}$ enhanced elastic and inelastic light scattering by $\mathrm{Si} \mathrm{NWs},{ }^{12}$ light extinction, ${ }^{13}$ enhanced light

${ }^{\text {a)} E m a i l: ~ j i m e n e z @ f m c . u v a . e s ~}$ emission, ${ }^{14-17}$ second harmonic generation, ${ }^{18}$ and optical annealing of Si NWs, ${ }^{19}$ among others.

Compared to either dielectric micro-resonators or metal cavities, semiconductor NWs present additional chances for engineering the optical resonances. In particular, their suitability for fabricating complex structures such as heterojunctions (HJs), quantum wells, and superlattices or selective doping should provide additional means to engineer the light/NW interaction. HJs are fundamental for most electronic and optoelectronic devices, such as sensors, solar cells, and thermoelectric devices. The proper characterization of the HJ optical properties is essential to improve the performance of optoelectronic devices.

Quantitative analysis of the interaction between the electromagnetic (EM) radiation and NWs has been carried out by the extension to NWs of the Lorenz-Mie theory, developed for the study of the electromagnetic behavior of resonant micro-spheres. ${ }^{20}$ In this theory, the NWs are described as infinitely long cylinders immersed in a homogeneous and isotropic non-absorbing medium. The calculation of the absorption and scattering efficiencies of NWs, $\mathrm{Q}_{\mathrm{abs}}$, and $\mathrm{Q}_{\mathrm{sc}}$, respectively, has revealed a strong dependence of these magnitudes with the NW diameter and the light wavelength. ${ }^{10}$ Numerical solutions of the problem by the finite difference in the time domain (FDTD) method showed that the electric field inside the NW is not homogeneously distributed, but a pattern of lobed like structures, whose distribution depends on the NW diameter, is observed. ${ }^{7}$ 
We study herein the interaction between a laser beam focused through a high magnification microscope objective and group IV semiconductor NWs, both compositionally homogeneous and axially heterostructured. This interaction is experimentally monitored by micro-Raman spectroscopy, and the results are contrasted with the solution of the Maxwell equations using finite element methods (FEM). In Sec. II, we describe the samples and the experimental procedure, Sec. III deals with the Raman analysis of these NWs, Sec. IV is devoted to the FEM modelling of the light/NW coupling, and, finally, Sec. V presents the discussion of the results, highlighting the role of the axial heterojunctions as optical enhancers.

\section{EXPERIMENTAL AND SAMPLES}

Group IV NWs were grown by the vapour-liquid-solid (VLS) method using a commercial low pressure chemical vapor deposition (LPCVD) reactor. Either $\mathrm{SiH}_{4}$ or $\mathrm{Si}_{2} \mathrm{H}_{6}$ and $\mathrm{GeH}_{4}$ were used as precursor gases, and Au metal droplets were used as catalysts. ${ }^{21}$

Single Si NWs were grown at $470{ }^{\circ} \mathrm{C}$ using $\mathrm{Si}_{2} \mathrm{H}_{6}$ as a precursor, while the SiGe NWs (with a nominal Ge atomic fraction close to 0.1 ) were grown at $430{ }^{\circ} \mathrm{C}$. The carrier gas was $\mathrm{H}_{2}$, preserving for the different precursor flow ratios a total pressure of 400 mTorr all over the growth run. ${ }^{21}$ Under these growth conditions, straight NWs with uniform diameters ranging from 30 to $100 \mathrm{~nm}$ were obtained. A crucial issue concerns the $\mathrm{HJ}$; in a previous work, the different growth approaches followed to achieve the matching between the $\mathrm{SiGe}$ and $\mathrm{Si}$ segments in the axially heterostructured NWs were discussed. ${ }^{22}$ The best way to achieve a good junction between $\mathrm{SiGe}$ and $\mathrm{Si}$ was by switching off the $\mathrm{GeH}_{4}$ source, while keeping a continuous flow of $\mathrm{Si}_{2} \mathrm{H}_{6}$.

The morphological features of the NWs were studied in a high resolution field emission scanning electron microscope (FESEM). The NWs lengths varied from 2 to $5 \mu \mathrm{m}$, and they appear as straight cylinders without appreciable tapering. Transmission Electron Microscopy (TEM) showed the excellent crystallinity of the NWs, Fig. 1(b). In particular, high resolution TEM images of the $\mathrm{HJ}$ region of $\mathrm{SiGe} / \mathrm{Si}$ HJs did not show structural discontinuities at the junction, nor stacking faults, or other structural defects. ${ }^{21,22}$

It is claimed that in VLS growth, the abruptness of the $\mathrm{HJ}$ depends on the solubility of the atomic species in the catalyst metal. Due to the reservoir effect in the catalyst droplet

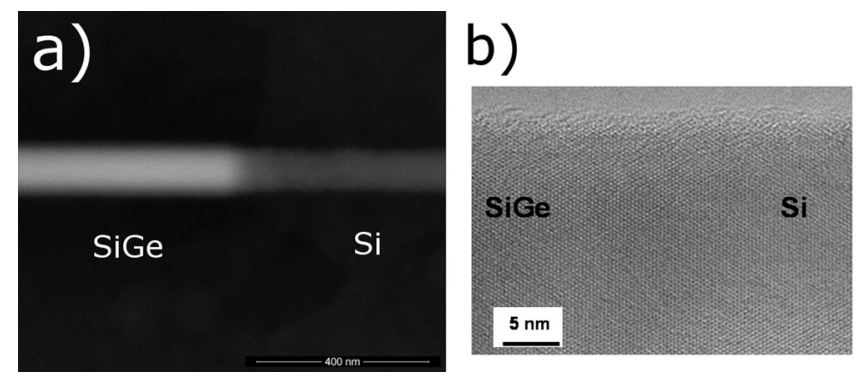

FIG. 1. (a) Backscattering SEM image of a heterostructured SiGe/Si NW (diameter $\approx 110 \mathrm{~nm}$ ). (b) HR-TEM image of the HJ region of a $\mathrm{SiGe} / \mathrm{Si}$ axially heterostructured NW showing the excellent crystalline quality of the transition and the absence of any kind of defects. once the precursor gas source is switched-off to shift to the composition of the next NW segment, the remaining atoms solved in the catalyst droplet continue to be deposited up to reaching a sub-saturation concentration. The result is a compositionally graded junction, with the composition varying between the compositions of the two NW segments, $\mathrm{Si}_{1-\mathrm{x}} \mathrm{Ge}_{\mathrm{x}}$ and $\mathrm{Si}$, respectively. This effect is particularly relevant in the SiGe system because of the high solubility of Ge in Au. According to this, the $\mathrm{SiGe} / \mathrm{Si} \mathrm{HJ}$ presents a characteristic trailing gradual composition. The trailing $\mathrm{HJ}$ width was claimed to be roughly equivalent to the NW diameter, ${ }^{23-25}$ and thus, the axially heterostructured NWs consist of a $\mathrm{Si}_{1-\mathrm{x}} \mathrm{Ge}_{\mathrm{x}}$ segment, a narrow $\mathrm{Si}_{1-\mathrm{y}} \mathrm{Ge}_{\mathrm{y}} \mathrm{HJ}$ layer with a graded $\mathrm{Ge}$ composition $(\mathrm{x} \geq \mathrm{y} \geq 0)$, and a $\mathrm{Si}$ segment. Atomicresolution high-angle annular dark-field scanning-transmission electron microscopy (HAADF-STEM) reveals the existence of the compositionally graded $\mathrm{HJ},{ }^{24}$ which can also be observed by high resolution energy dispersive X-Ray spectroscopy (EDS), though a precise estimation of the composition gradient is not easy to be achieved because of the e-beam size.

As-grown NWs were separated from the substrate in an ultrasonic bath and suspended in methanol. Droplets of this suspension were spread out on an $\mathrm{Al}$ coated $\mathrm{Si}$ substrate for the micro-Raman $(\mu-\mathrm{R})$ measurements. The choice of the substrates is based on the optimization of the Raman signal, which is enhanced by the presence of the metallic substrate with respect to free standing NWs. Also, but not less important, the presence of the metallic substrate improves the thermal management of the NW allowing to minimize the laser induced heating during the $\mu-\mathrm{R}$ experiments. $\mathrm{Al}$ coated substrates were satisfactory from both the thermal point of view and the enhancement of the Raman signal. Therefore, they were used as the standard substrate for our micro-Raman measurements. The Al coating was around $0.5 \mu \mathrm{m}$ thick, enough to block any optical signal arising from the Si substrate. This thickness also guarantees a continuous $\mathrm{Al}$ layer with no $\mathrm{Al}$ islands, ruling out any $\mathrm{Al}$ related plasmon enhancement of the Raman signal.

$\mu$-R spectra were recorded with a Labram UV-HR 800 Raman spectrometer from Horiba-Jobin Yvon. The excitation and the scattered light collection were performed by means of a confocal metallographic microscope with a high magnification objective $(\times 100)$ and a numerical aperture of 0.95 . A frequency doubled Nd:YAG laser $(532 \mathrm{~nm})$ was used as the excitation source. The laser beam diameter at the focal plane is slightly below $1 \mu \mathrm{m}$ according to the Abbe formula $(\phi=1.22 \lambda / \mathrm{NA})$, which is several times larger than the diameter of the studied NWs, which typically ranges from 30 to $100 \mathrm{~nm}$. It is important to note that the effective excitation power on the NW depends on its position inside the Gaussian power distribution of the focused laser beam. ${ }^{26,27}$ Prior to the Raman measurements, the dimensions and morphology of each NW were characterized in a field emission scanning electron microscope (FESEM).

\section{EXPERIMENTAL RESULTS}

$\mu$-Raman spectroscopy has demonstrated to be a powerful non-destructive technique for the characterization of semiconductor NWs. It provides information about several relevant aspects of the NWs, e.g., the structure, composition, 


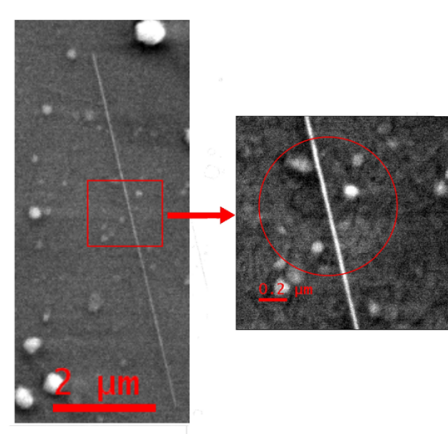

(a)

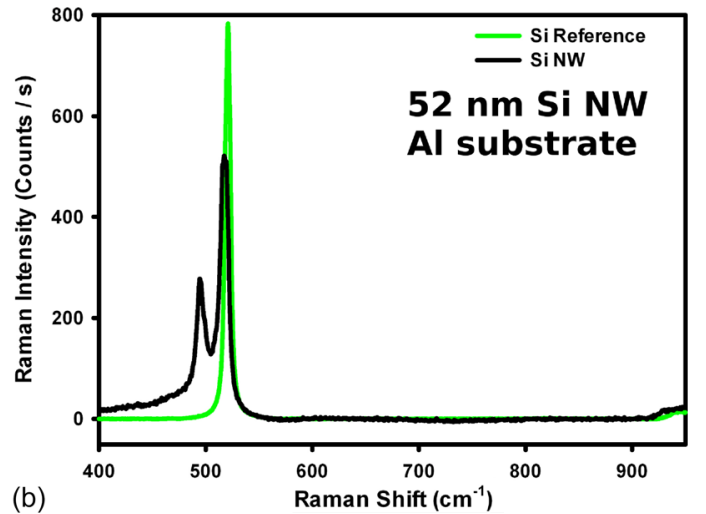

FIG. 2. (a) SEM image of a $\mathrm{Si} \mathrm{NW}$ $(52 \mathrm{~nm}$ diameter). The red circle represents the laser beam spot. (b) Raman spectrum of a bare $\mathrm{Si}$ substrate and a $\mathrm{Si}$ $\mathrm{NW}$ on an $\mathrm{Al}$ substrate, respectively, showing comparable intensities in spite of the different scattering volumes. The low frequency peak at $495 \mathrm{~cm}^{-1}$ observed in the Si NW is associated with either a surface phonon or a hexagonal polytype. Its presence does not alter the course of the discussion, and the debate about it is out of the scope of this work. stress, and thermal, electronic, and optical properties. ${ }^{5,7,28-32}$ Some of the above-mentioned properties depend on the NW dimension. Therefore, in order to give sense to the Raman spectrum, one needs to work with individual NWs instead of ensembles of NWs, which give a Raman spectrum averaged over a certain population of NWs with a distribution of diameters and lengths. Furthermore, the Raman signal is proportional to the excitation light intensity (i.e., $\propto|\mathrm{E}|^{2}$ ), which makes Raman scattering an excellent tool to catch sight of the electromagnetic interaction between NWs and light.

A priori, the measurement of the Raman spectrum of individual NWs is challenging because of the low efficiency of Raman scattering, which is a second order optical process. This results in a weak Raman signal when dealing with small sampling volumes, as it can be the case of individual NWs. On the other hand, NWs are not efficient thermal carriers; ${ }^{2,3}$ therefore, in order to avoid laser induced heating, one has to keep low excitation laser power densities. Laser heating might modify the shape of the Raman spectrum leading to misinterpretations; in particular, free standing NWs are substantially heated up during Raman experiments because of the poor heat dissipation across them. ${ }^{2,3,26,27,31}$ Laser induced heating is almost negligible under our experimental conditions because of the use of a metallic substrate, namely, $\mathrm{Al}$ coated $\mathrm{Si}$, and the adequate excitation conditions.

When the NWs are deposited on certain substrates, e.g., $\mathrm{Al}$ coated $\mathrm{Si}$, they can be directly observed at the optical microscope in spite of their deeply subwavelength diameter, which evidences that the NWs behave as nanolenses for visible light. Furthermore, the observation of the NWs in the optical microscope depends on its orientation with respect to the light polarization axis, showing that the NW is a very anisotropic optical object. ${ }^{16,17}$

All these optical behaviors point to an unusual interaction between the NW and light. This is well observed when one measures the Raman spectrum of individual Si NWs. Regardless of the small sampling volume, when the NWs were deposited on different substrates, the Raman intensities recorded for individual NWs reached the same order of magnitude as the intensity recorded for bulk $\mathrm{Si}$ under similar excitation conditions, Fig. 2. Note that in terms of Raman intensity per unit of scattering volume, the Raman signal arising from the NW presents a significant enhancement with respect to the bulk Si signal, about two orders of magnitude. The NWs behave as optical nano-antennas rendering the
Raman spectrum of individual NWs measurable under low laser power excitation for resonant diameters. ${ }^{28-30}$ Therefore, $\mu-\mathrm{R}$ spectra of individual NWs without external perturbations, e.g., heating, can be recorded if one adopts the appropriate experimental configuration.

As mentioned above, the NWs are optically anisotropic, and as a result, the Raman signal will strongly depend on the polarization of the incident light. A polar plot of the recorded Raman intensity with respect to the angle formed between the light polarization and the NW axis will show a dipolar behavior in which the maximum signal is observed when the electric field of the incident light is aligned parallel to the NW axis, Fig. 3. All the Raman spectra of the NWs presented here were recorded in this configuration to optimize the signal/noise ratio.

The full characterization of the NWs was carried out acquiring the Raman spectra by scanning the laser beam either along or across the NW axis in steps of 50-100 nanometers. Transverse laser scans across the NW permit us to establish the Raman response of the NW to the laser intensity. In previous works, we studied the effective excitation intensity depending on the position of the NW inside the Gaussian power distribution of a focused laser beam. ${ }^{26,27}$ When the Raman peak frequency remains unchanged across

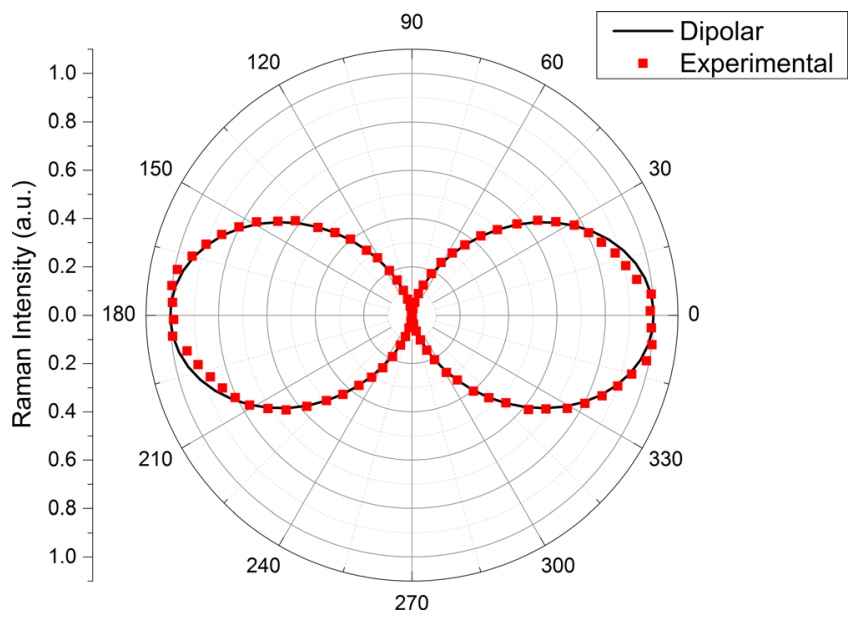

FIG. 3. Polar plot of the normalized Raman intensity of the HJ region of a $\mathrm{Si} / \mathrm{SiGe}$ heterostructured NW recorded for different polarization angles of the incident light with respect to the NW axis. The plot shows a clear dipolar behaviour, and the result is the same for all Raman signals coming from the NW. 
the transverse scan, one can assume that there is not noticeable heating of the NW by the laser beam for that selected laser power. The Raman peak frequencies obtained from the transverse scan across a Si NW are shown in Fig. 4(b). One observes that there is a negligible frequency shift along the scanning line, which means that the NW is not heated by the laser beam within the range of powers drawn by the laser power Gaussian profile, see the temperature conversion in Fig. 4(c). When the laser power increases, one observes the typical peak frequency downshift and broadening associated with increased temperature. Therefore, taking into account the temperature profiles of Fig. 4, one can ascertain that for laser powers below $50 \mu \mathrm{W}(532 \mathrm{~nm})$, one can work in conditions of negligible laser induced heating. Therefore, transverse scans across the NW are suitable for fixing the optimal excitation conditions and also permit us to align the NW with the beam axis.

\section{A. Axially heterostructured NWs}

Up until now, most of the research interest about the light/NW interaction has focused on homogeneous single $\mathrm{NWs},{ }^{5,6,10}$ while the role of the heterojunctions in the light/ NW interaction has been scarcely studied and mainly focused on core/shell HJs. ${ }^{33}$

The analysis of the axially heterostructured NWs was done by scanning the laser beam along the NW axis in steps of $100 \mathrm{~nm}$. As mentioned above, the trailing SiGe/Si HJ consists of a compositionally graded layer with a thickness roughly equivalent to the NW diameter. ${ }^{22-25,34}$ A priori, when the focused laser beam shares the two NW segments and the HJ, the Raman signal arising from the HJ layer must be much less intense than the Raman signals arising from the two NW segments, in terms of scattering volume figures. Under normal conditions, it should be scarcely detected, because of the very small sampling volume of the HJ. The sampling volumes of the three NW regions simultaneously shared by the laser beam, $\mathrm{Si}_{1-\mathrm{x}} \mathrm{Ge}_{\mathrm{x}} / \mathrm{Si}_{1-\mathrm{y}} \mathrm{Ge}_{\mathrm{y}}(\mathrm{HJ}) / \mathrm{Si}$, obey to a ratio $\approx 1: 0.08: 1$ for a $40 \mathrm{~nm}$ diameter $\mathrm{NW}$, assuming a $\mathrm{HJ}$ width equivalent to the NW diameter. Once the volume ratio is corrected by the Gaussian laser power distribution, assuming that the $\mathrm{HJ}$ is placed in the laser beam center, the integrated laser power ratio for the three sampled volumes scales to $\approx 1: 0.1: 1$, which is still greatly unfavorable for the $\mathrm{HJ}$ region in terms of effective excitation.

Typical Raman spectra (only the Si-Si Raman mode of the SiGe alloy is shown) obtained along a $50 \mathrm{~nm}$ diameter axially heterostructured $\mathrm{SiGe} / \mathrm{Si} \mathrm{NW}$ deposited on an Al coated Si substrate can be seen in Fig. 2 of Ref. 39. One observes the typical Raman spectrum of a $\mathrm{Si}$ NW for the laser beam focused on the $\mathrm{Si}$ segment. When it is focused on the $\mathrm{Si}_{1-\mathrm{x}} \mathrm{Ge}_{\mathrm{x}}$ segment, one observes the Raman spectrum of the SiGe alloy with the nominal Ge composition, which in this case is $\approx 10 \%$. This has been confirmed by the peak frequency shift of the Si-Si Raman mode ${ }^{35,36}$ and also by EDS measurements in the TEM. Note that the studied NWs have diameters (>30 nm) for which phonon confinement does not occur, ${ }^{37,38}$ and then, Raman bands are not disturbed by size effects. Regarding the Raman spectrum recorded when the laser beam shares the two NW segments and the HJ, one observes a band peaking at an intermediate frequency between the ones of the $\mathrm{Si}$ and $\mathrm{Si}_{1-\mathrm{x}} \mathrm{Ge}_{\mathrm{x}} \mathrm{NW}$ segments. While the spectra taken in the single NW segments are fitted by only one Lorentzian curve, the spectrum sharing the HJ needs three Lorentzian curves to be satisfactorily fitted. Each of the three bands resulting from the deconvolution corresponds to the $\mathrm{Si}_{1-\mathrm{x}} \mathrm{Ge}_{\mathrm{x}}$ segment, the $\mathrm{Si}$ segment, and the HJ layer itself, respectively. In the case of the HJ, the Raman spectrum looks like the SiGe spectrum corresponding to an intermediate composition in between those of the two homogeneous segments, see Fig. 3 of Ref. 39. Surprisingly, the Raman intensity arising from the $\mathrm{HJ}$ is as intense as, or even higher than, the Raman intensities arising from the two NW segments, instead of the expected 1:0.1:1 volume ratio reported previously. Therefore, a significant Raman enhancement, at least 10 to 20 times, shall be localized at the HJ, which points to a significant local electromagnetic enhancement in the very $\mathrm{HJ}$ region. ${ }^{39}$

This is better observed in a $\mathrm{Si}_{1-\mathrm{x}} \mathrm{Ge}_{\mathrm{x}} / \mathrm{Si}$ axially heterostructured NW with a higher Ge content, $\mathrm{x} \approx 0.6$ in this
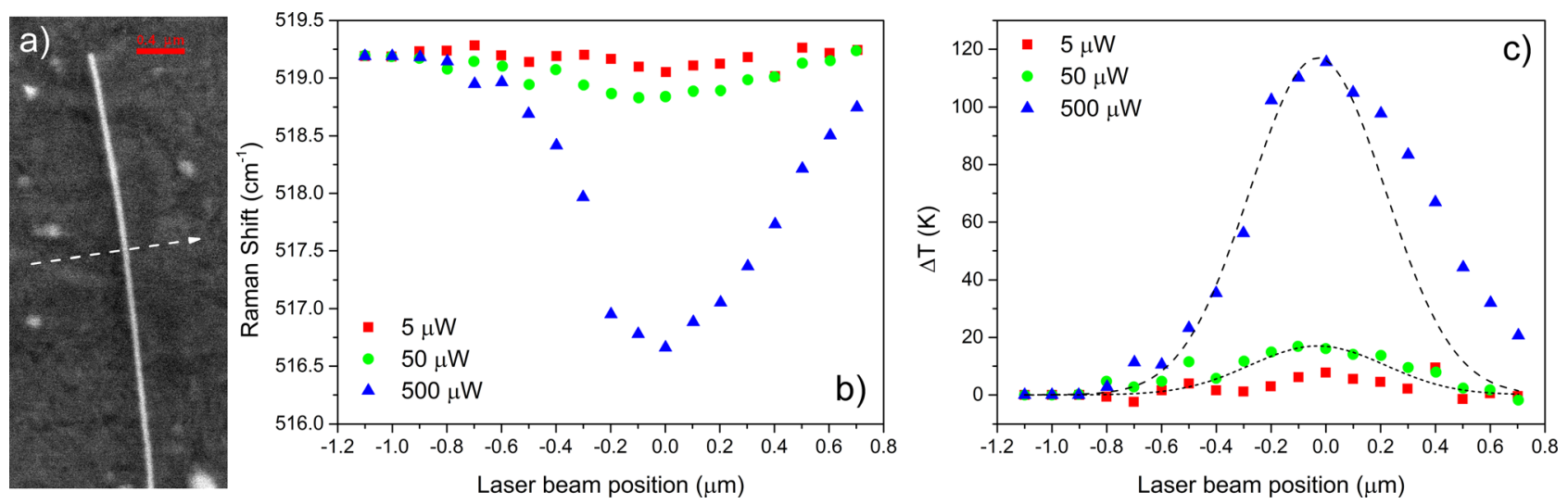

FIG. 4. (a) SEM image of a Si NW. The arrow indicates the scanning line crossing the NW, (b) Raman shift profiles for different excitation conditions, see the symbols on the right side of the figure. (c) Estimated temperature deduced from the Raman shift. Note that as the NW position is closer to the beam axis, the effective excitation laser power density is enhanced because of the Gaussian laser power distribution. One observes that laser powers up to $50 \mu \mathrm{W}$ ( $532 \mathrm{~nm}$ ) permit us to work without significant NW heating, while for higher laser powers, the temperature starts to increase under the laser exposure. Because of the Gaussian power distribution, the effective excitation laser power depends on the position of the NW with respect to the laser beam axis. These scans permit us to fix the conditions for which the temperature enhancement can be neglected. 
case. $^{40}$ The larger shift of the Si-Si peak of the SiGe alloy permits us to observe the different contributions with minimum overlapping. In these NWs, a dramatic spectral change occurs when the laser beam crosses the HJ region, Fig. 5. One observes a change in the relative intensities and a shift of the peak frequencies of the Raman bands of the SiGe alloy, both clear evidences of compositional changes at the $\mathrm{HJ}$ with respect to the two NW segments. Once again, the spectrum collected at the HJ, identified through its Raman spectrum, shows a much higher intensity than it should correspond to its volume, Fig. 5.

The relevant point is that the Raman spectrum of the HJ is clearly seen in spite of the small scattering volume associated with the HJ. For these NWs, the measured Raman intensity per unit volume arising from the $\mathrm{HJ}$ is enhanced by a factor of about 60 with respect to the Raman signal collected in the pure NW segments. This evidences that the HJ provides an additional contribution to the coupling between the laser beam and the NW. In other words, the axial HJ concentrates the electromagnetic field under visible illumination, which appears very suitable for photonic applications, e.g., photon harvesting in solar cells or photon detectors.

Note that this is not a mere compositional effect. In fact, the Raman signal of $\mathrm{Si}_{1-\mathrm{x}} \mathrm{Ge}_{\mathrm{x}}$ with $\mathrm{x}=0.7-0.8$ presents a resonance for $532 \mathrm{~nm}$ excitation. ${ }^{41}$ This resonance is progressively quenched for decreasing $\mathrm{x}$; therefore, if we were observing a simple compositional resonance, the Raman spectrum collected from the SiGe segment $(x \approx 0.6)$ will supply a higher intensity than that from the HJ, where the value
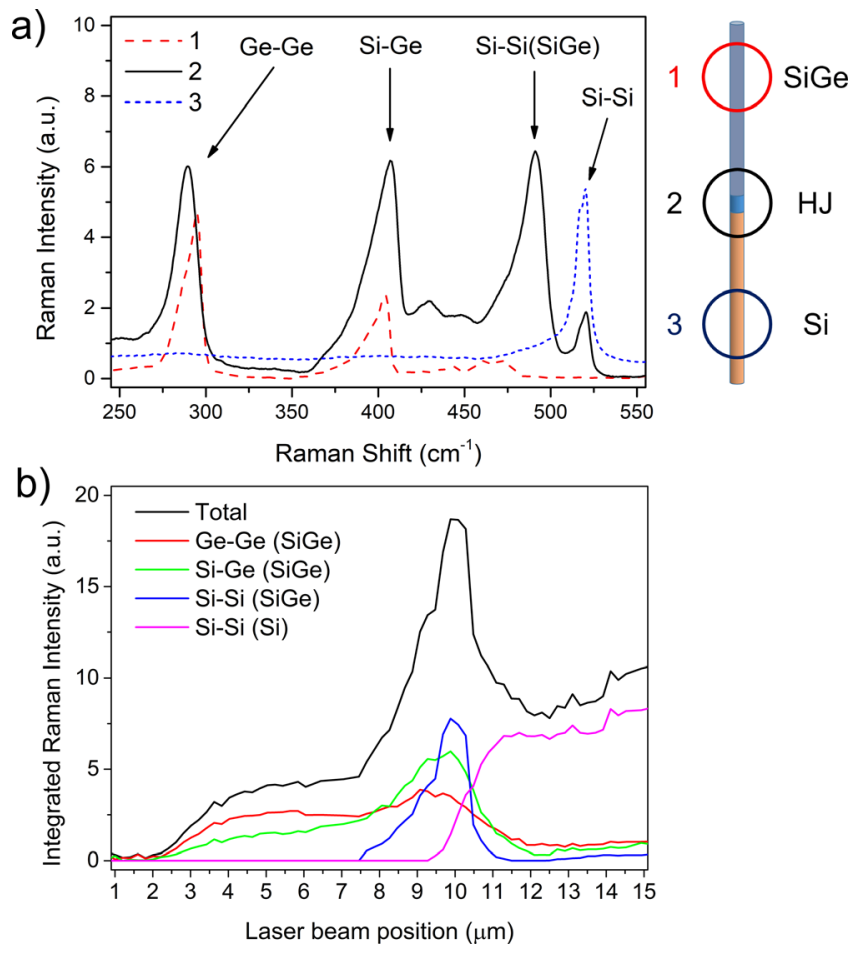

FIG. 5. (a) Raman spectra in $\mathrm{SiGe} / \mathrm{Si}$ heterostructured NW $100 \mathrm{~nm}$ in diameter with a nominal Ge concentration of $\approx 60 \%$. The dramatic changes in the spectrum when the $\mathrm{HJ}$ is probed provide evidence of the composition change. (b) Raman intensity profiles of the Raman bands of $\mathrm{Si}$ and $\mathrm{SiGe}$ as a function of the laser beam position along the NW, showing the enhancement of the Raman signal at the HJ. of $\mathrm{x}$ progressively decreases, being out of compositional resonance. It is worth mentioning that the Raman signal arising from the $\mathrm{HJ}$ of the heterostructured $\mathrm{Si} / \mathrm{Ge} \mathrm{NWs}$ was also observed by Wang et al. ${ }^{42}$

\section{MODELLING OF THE LASER/NW INTERACTION}

The understanding of the light/NW interaction demands the solution of the Maxwell equations of the system formed by the laser beam, the NW, and the hosting medium. The experimental conditions must be carefully considered when one acquires the spectrum of a NW with a micro-Raman apparatus (far field). First, the laser beam is focused by a high magnification optical microscope objective with a large numerical aperture, which gives a focused Gaussian-like laser intensity distribution. Second, as mentioned in Secs. II and III, the NWs have a finite length and an optical discontinuity due to the HJ. Finally, the NWs can be either free standing surrounded by air or lying on a substrate. All of this shall be consider in the modelling.

The problem of the interaction of the EM wave and the NW is usually studied in terms of the Mie scattering theory. For this, an ideal homogeneous and infinitely long NW is considered, neglecting the influence of the NW ends. Most of the simulations rely on the illumination with polarized plane waves, while only a few studies have considered focused laser beams. ${ }^{7}$ The presence of the NW ends and the HJ is not compatible with the infinite and homogeneous NW of the Mie theory. In this work, Maxwell equations in 3-dimensional space were solved using the Electromagnetic Waves in Frequency Domain module of COMSOL Multiphysics.

In the simulation, a heterostructured NW is placed on the substrate, which fills the inferior half-space, while the space surrounding the NW is set up to be air. The laser beam is described as a focused Gaussian beam, instead of the usual approach of a plane wave. In this situation, the distribution of the EM field inside the NW is far from what a homogeneous illumination would induce, and the plane wave approximation is no longer valid.

The equations accounting for the electric field in a Gaussian laser beam propagating along the $\mathrm{z}$ axis are written as follows:

$$
\begin{gathered}
E_{x}(r, z, t)=E_{0} \frac{w_{0}}{w(z)} e^{\left(\frac{-r^{2}}{w^{2}(z)}\right)} e^{\left(i k \frac{r^{2}}{2 R(z)}\right)} e^{i(k z-\omega t)} e^{i \zeta(z)}, \\
w(z)=w(0) \sqrt{1+\left(\frac{z \lambda}{\pi w_{0}^{2}}\right)} \\
R(z)=z\left(1+\left(\frac{\pi w_{0}^{2}}{z \lambda}\right)^{2}\right) \\
\zeta(z)=\arctan \left(\frac{z \lambda}{\pi w_{0}^{2}}\right)
\end{gathered}
$$

and cylindrical coordinates are used: $\mathrm{r}$ is the distance to the beam axis ( $\mathrm{z}$ axis) and $\mathrm{z}$ the position with respect to the focal plane $(z=0)$. $R(z)$ is the radius of curvature of the wavefront at $\mathrm{z}, \zeta(\mathrm{z})$ is called the Guoy phase at $\mathrm{z}$, typical of Gaussian 
and spherical wavefronts, and $w(z)$ is the diameter of the Gaussian spot at $\mathrm{z}$, which depends on its value at the focus $\mathrm{w}_{0}=\mathrm{w}(0)$, the so called waist size. Let us remind that the $\mathrm{NW}$ is lying on the $\mathrm{x}-\mathrm{y}$ plane.

According to Abbe's formula, the beam waist size, $\mathrm{w}_{0}$, is determined by the laser wavelength and the numerical aperture of the objective

$$
w_{0} \propto \frac{1.22 \lambda}{N A} .
$$

Once the excitation laser beam and the system have been modelled, the FEM software solves the equations of the scattered field. The scattered field, $\mathrm{E}_{\mathrm{sc}}$, is defined as the EM field generated by the system when the excitation field, $\mathrm{E}_{\mathrm{b}}$, is applied, in order to create a total EM field satisfying the Maxwell equations

$$
\vec{E}_{t o t}=\vec{E}_{b}+\vec{E}_{s c} \leftrightarrow \vec{E}_{s c}=\vec{E}_{t o t}-\vec{E}_{b}
$$

When the distribution of the total EM field inside the NW is known, the expected Raman signal of each segment of the NW can be calculated since the emitted Raman signal is proportional to the EM field intensity, $I_{R} \propto|E|^{2}$. Therefore, once we have calculated the distribution of the electric field inside the NW, the Raman signal arising from a certain region of the NW will be proportional to the integral of $|\mathrm{E}|^{2}$ over the corresponding sampling volume. By computing these integrals, we can plot the corresponding theoretical Raman signal along the NW.

Finally, the model is solved for different positions of the laser beam along the NW axis in order to reproduce the experimental longitudinal scans of the NW. This allows us to calculate the Raman intensities of the NW as a function of the laser beam position. The optical parameters used for the calculations are resumed in Table I and were extracted from Sopra database. ${ }^{43}$

\section{DISCUSSION}

The tunability of the optical absorption by NWs requires the control of the different factors contributing to the enhancement of the optical absorption/scattering. The FEM model permits us to analyze the impact of the enhancement factors, namely, the NW dimension, the substrate, the light wavelength and polarization, and the NW nature and structure.

We solved the problem for different substrates characterized by different complex refractive indexes. In particular, the results obtained for $\mathrm{Au}, \mathrm{Al}, \mathrm{Ge}$, and air are shown in Fig. 6 for the $532 \mathrm{~nm}$ wavelength. This figure shows the diameter dependent resonances for $|\mathrm{E}|^{2}$. If the integrated $|\mathrm{E}|^{2}$ is plotted as a

TABLE I. Real (n) and imaginary (k) parts of the complex refractive indexes of the different materials used in the FEM calculations for $532 \mathrm{~nm}$ radiation. $^{43}$

\begin{tabular}{cccccc}
\hline \hline Au & Al & Si & Ge & Si(1-x)Gex \\
\hline n & 0.467 & 0.93877 & 4.1334 & 4.92436 & $4.1334+0.668619 x+1.510779 x^{2}$ \\
$\mathrm{k}$ & 2.407 & 6.4195 & 0.033258 & 2.3734 & $0.033258-0.204615 x+1.621028 x^{2}$ \\
\hline \hline
\end{tabular}
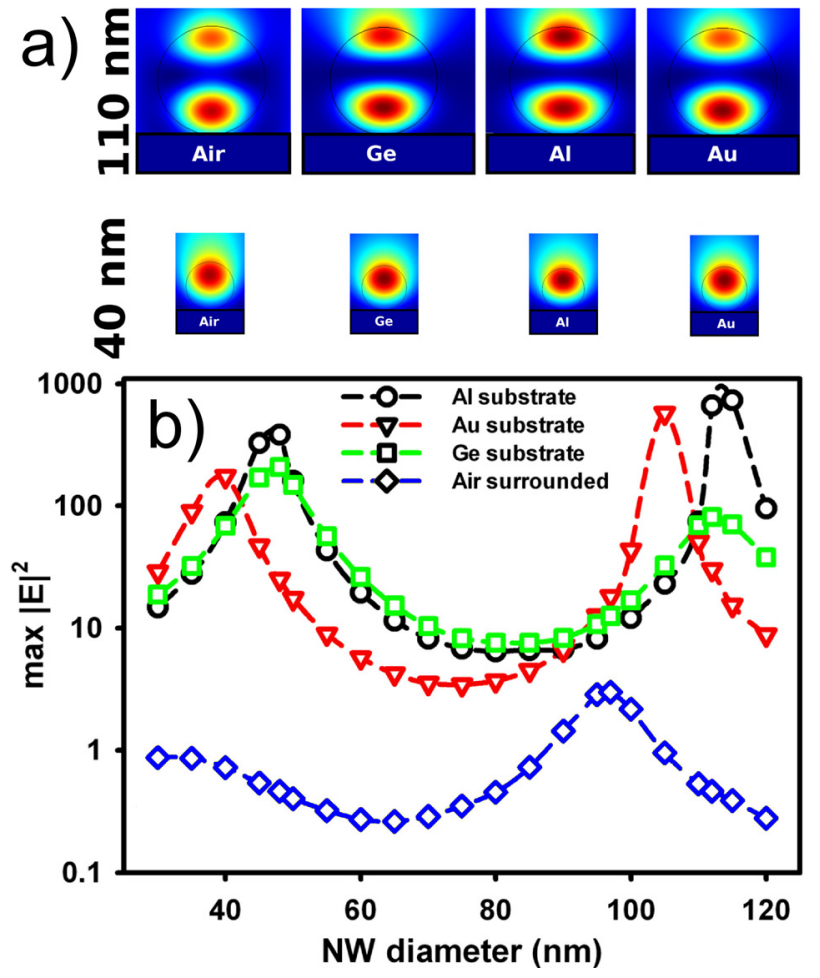

FIG. 6. (a) Electromagnetic field distributions for two Si NWs with diameters of 40 and $110 \mathrm{~nm}$, with different NW/substrate configurations. (b) $|\mathrm{E}|^{2}$ vs NW diameter for the different configurations.

function of the NW diameter, one observes the enhancement of $|E|^{2}$, up to one order of magnitude for the NWs standing in air with respect to the incident electromagnetic field and up to 2 additional orders of magnitude for the NWs lying on the metallic substrate. The reflection of both the excitation and the Raman radiation by the metallic substrate can contribute to this enhancement by a theoretical factor of 4 , and the remaining amplification is due to the EM interaction between light and the NW-substrate system. The substrate acts by improving the antenna effect already present in free standing NWs. Figure 6 also shows that the resonance diameters depend on the substrate. There is a shift of the resonances to lower diameters for $\mathrm{Au}$ with respect to $\mathrm{Al}$ and a further shift to lower diameters for the free standing NW in air, as it was already shown in Ref. 7 using FDTD calculations. The resonances for the Ge substrate closely match those of the Al substrate but with lower electromagnetic enhancement. According to this, the optical response of the NWs can be tuned by using different diameters and substrates characterized by different optical constants.

\section{A. Axial heterojunctions}

The study of axial heterostructures demands a 3D solution of the Maxwell equations in order to reveal the role of the HJ. We have solved the Maxwell equations for a $\mathrm{NW}$ with a $\mathrm{SiGe} / \mathrm{Si}$ axial $\mathrm{HJ}$ interacting with the focused Gaussian laser beam. The 3D solution of the Maxwell equations reveals the enhancement of the electric field in the HJ region, in agreement with the Raman measurements, Fig. 7. The 3D simulation also reveals that the electromagnetic field profile along the NW axis exhibits a series of longitudinal 


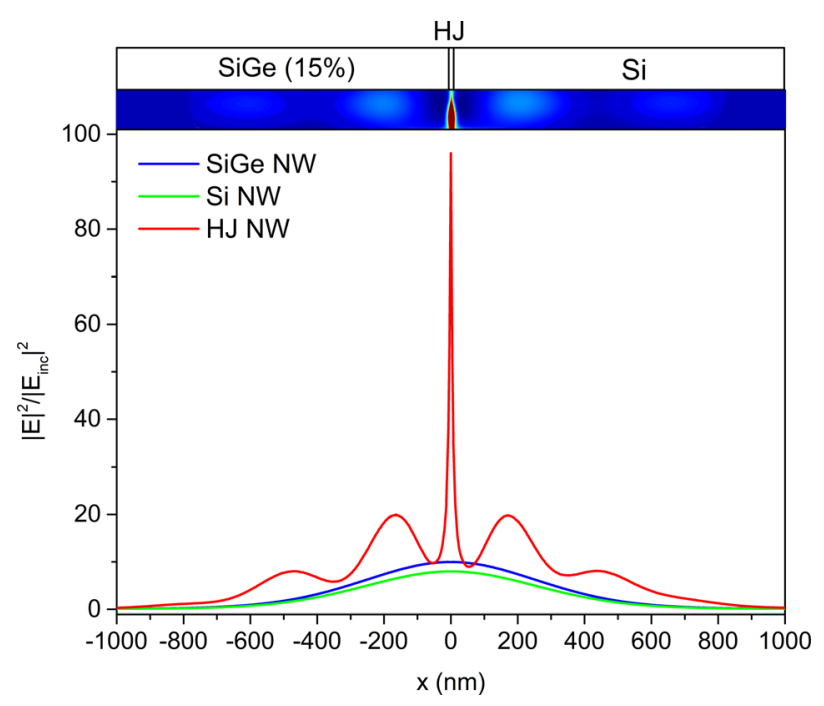

FIG. 7. Profiles of the normalized value of $|E|^{2}$ along the HJ NW axis under a focused Gaussian beam excitation, showing the enhancement at the HJ. The response of single $\mathrm{Si}$ and SiGe NWs is also plotted for comparison. The simulated NW diameter is $40 \mathrm{~nm}$. The heterostructured NW shows a few longitudinal modes, which are scarcely appreciated in the Si NW.

resonant modes overlapped with the Gaussian distribution. These longitudinal modes can be clearly seen when a plane wave excitation is considered on a pure Si NW of finite length, but they are hidden by the Gaussian beam distribution in the local excitation case. However, in the presence of the HJ, they are clearly revealed, evidencing that the HJ produces a significant disturbance of the electromagnetic field inside the NW. In addition, the HJ increases the electromagnetic field intensity inside the NW, and this overall enhancement is also experimentally observed in the Raman scans along the NW axis; in Fig. 5(b), a higher Raman intensity is observed in the presence of the HJ.

In order to confirm that the $|\mathrm{E}|^{2}$ resonance at the $\mathrm{HJ}$ is related to the presence of the $\mathrm{HJ}$ itself and it is not an effect of the mere difference in the resonance behaviour between

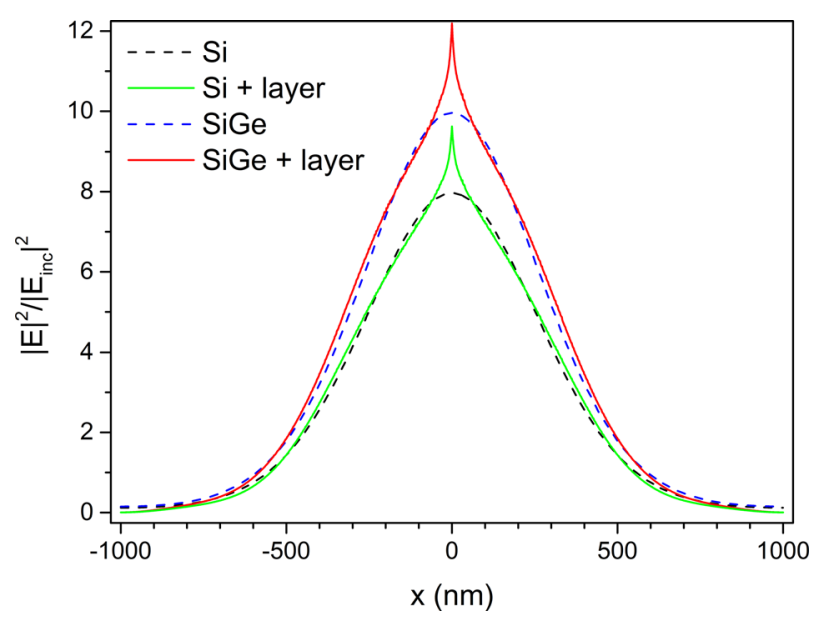

FIG. 8. Relative electromagnetic field intensity distribution inside $\mathrm{Si}$ and SiGe ( $15 \% \mathrm{Ge})$ NWs (40 diameter $\mathrm{nm}$ ), black and blue dashed lines. Green and red lines represent the same magnitude for the same NWs with an axial thin layer $(6 \mathrm{~nm})$ of $\mathrm{SiGe}(7.5 \% \mathrm{Ge})$. The figure shows how the presence of a different material enhances the EM field in its vicinity, regardless of the Ge composition difference. the two NW segments, we modelled two different scenarios for a $40 \mathrm{~nm}$ diameter NW. The first one is a pure Si NW with a $\operatorname{SiGe}(x=0.075)$ thin slab $(6 \mathrm{~nm}$ width $)$ in its center, and the second one is a pure $\operatorname{SiGe}(x=0.15)$ with the same SiGe $(x=0.075)$ slab. If we were dealing with a mere compositional effect, both cases should exhibit opposite behaviours in the HJ region. However, the local enhancement of the electric field at the HJ is observed for both NW configurations, which points to the electromagnetic field enhancement by the presence of the HJ, Fig. 8.

Finally, the calculated enhancement factor of $|\mathrm{E}|^{2}$ at the $\mathrm{HJ}$ of a Si/SiGe NW with the same structure as the experimentally measured ones is 63. This value is in very good agreement with the mean value of 60 experimentally measured by $\mu$-Raman spectroscopy.

\section{CONCLUSIONS}

An exhaustive analysis of the NW/light coupling has been carried out combining electromagnetic modelling and micro-Raman experiments on individual NWs, aiming to understand the light/NW interaction. The Raman experiments evidenced different optical enhancement phenomena, some of them already known, while others as the enhancement associated with the axial HJs are new. The Raman experiments have demonstrated the possibility of tuning the light/NW interaction by varying different configurations of the NWs, namely, diameter, substrate, length, composition, and NW structure, in particular, the presence of HJs. A description of different micro-Raman experiments on NWs revealing the different optical effects is provided.

Numerical solutions of the Maxwell equations for the different experimental configurations of the light/NW system have been carried out. In particular, the laser beam/heterostructured NW interaction has been numerically analyzed by 3-D finite difference solutions of the Maxwell equations. Furthermore, instead of an infinite plane wave, a focused laser beam with a Gaussian intensity distribution has been used, more accorded with the comparison to the microRaman experiment. Additionally, we have considered different substrates supporting the NWs, since the resonance properties of the NWs depend on the electromagnetic properties of the surrounding medium as well.

The results presented herein show different possibilities for tuning the optical properties of the NWs. In addition to the diameter and composition dependence of the optical properties of the NWs, further consideration must be given to the NW length, the presence of HJs, and the surrounding medium. All of them would provide suitable means for tailoring the optical absorption/scattering of semiconductor NWs. This locally enhanced EM absorption might open interesting perspectives for photon engineering with heterostructured NWs; in particular, it seems very promising for photovoltaics and light detectors.

\section{ACKNOWLEDGMENTS}

This work was funded by Junta de Castilla y León (Projects VA293U13 and VA081U16) and Spanish Government (CICYT MAT2010-20441-C02 (01 and 02) and 
ENE 2014-56069-C4-4-R). J. L. Pura was granted by the FPU programme (Spanish Government) (FPU14/00916).

${ }^{1}$ G. Bronstrup, N. Jahr, A. Csaki, W. Fritzsche, and S. Chistiansen, ACS Nano 4, 7113 (2010).

${ }^{2}$ D. Li, Y. Wu, P. Kim, L. Shi, P. Yang, and A. Majumdar, Appl. Phys. Lett. 83, 2934 (2003).

${ }^{3}$ J. Anaya, T. Rodríguez, and J. Jiménez, Sci. Adv. Mater. 7, 1097 (2015).

${ }^{4}$ M. Law, D. J. Sirbuly, J. C. Johnson, J. Goldberger, R. J. Saykally, and P. Yang, Science 305, 1269-1273 (2004).

${ }^{5}$ H. Kallel, A. Arbouet, G. Bensssayag, A. Chehaidar, A. Potié, B. Salem, T. Baron, and V. Paillard, Phys. Rev. B 86, 085318 (2012).

${ }^{6}$ L. Cao, P. Fan, A. P. Vasudev, J. S. White, Z. Yu, W. Cai, J. A. Schuller, S. Fan, and M. L. Brongersma, Nano Lett. 10, 439 (2010).

${ }^{7}$ F. J. Lopez, J. K. Hyun, U. Givan, I. S. Kim, A. L. Holsteen, and L. J. Lauhon, Nano Lett. 12, 2266 (2012).

${ }^{8}$ L. Cao, J. S. White, J. S. Park, J. A. Schuller, B. M. Clemens, and M. L. Brongersma, Nat. Mater. 8, 643 (2009).

${ }^{9}$ R. Ruppin, Opt. Soc. Am. 15, 1891 (1998).

${ }^{10}$ G. S. Doerk, C. Carraro, and R. Maboudian, ACS Nano 4, 4908-4914 (2010).

${ }^{11}$ M. Heiss and A. Fontcuberta, Appl. Phys. Lett. 99, 263102 (2011).

${ }^{12}$ L. Cao, P. Fan, E. S. Barnard, A. M. Brown, and M. L. Brongersma, Nano Lett. 10, 2649 (2010).

${ }^{13}$ J. A. Schuller, R. Zia, T. Tauber, and M. L. Brongersma, Phys. Rev. Lett. 99, 107401 (2007).

${ }^{14}$ T. Nobis, E. M. Kaidas, A. Rahm, M. Lorenz, and M. Grundmann, Phys. Rev. Lett. 93, 103903 (2004).

${ }^{15}$ D. van Dam, D. R. Abujetas, R. Paniagua-Domínguez, J. A. Sanchez-Gil, E. P. A. M. Bakkers, J. E. M. Havekort, and J. Gómez-Rivas, Nano Lett. 15, 4557 (2015).

${ }^{16}$ H. E. Ruda and A. Shik, J. Appl. Phys. 100, 024314 (2006).

${ }^{17}$ H. E. Ruda and A. Shik, Phys. Rev. B 72, 115308 (2005).

${ }^{18}$ R. Grange, G. Brönstrup, M. Kiometzis, A. Sergeyev, J. Richter, C. Leiterer, W. Fritzsche, C. Gutsche, A. Lysov, W. Prost, F. Tegude, T. Pertsch, A. Tünnermann, and S. Christiansen, Nano Lett. 12, 5412 (2012).

${ }^{19}$ G. H. Ding, C. T. Chan, Z. Q. Zhang, and P. Sheng, Phys. Rev. B 71, 205302 (2005).

${ }^{20} \mathrm{M}$. Kerker, The Scattering of Light and Other Radiationsm (Academic Press, New York, USA, 1969).

${ }^{21}$ A. Rodríguez, J. Sangrador, T. Rodríguez, C. Ballesteros, A. C. Prieto, and J. Jiménez, Symposia $P / Q / R$ - Low-Dimensional Functional Nanostructures-Fabrication, Characterization and Applications (Mater. Res. Soc. Symp. Proc., 2010), Vol. 1258, P05-05.
${ }^{22}$ A. Rodríguez, T. Rodríguez, C. Ballesteros, and J. Jimenez, Symposium $D D$ - Group IV Semiconductor Nanostructures and Applications (Mater. Res. Soc. Symp. Proc., 2013), Vol. 1510, DD06-05.

${ }^{23}$ P. Periwal, N. V. Sibirev, G. Patriarche, B. Salem, F. Bassani, V. G. Dubrovskii, and T. Baron, Nano Lett. 14, 5140 (2014).

${ }^{24}$ T. E. Clark, P. Nimmatoori, K. K. Lew, L. Pan, J. M. Redwing, and E. C. Dickey, Nano Lett. 8, 1246 (2008).

${ }^{25}$ D. E. Perea, N. Li, R. M. Dickerson, A. Misra, and S. T. Picraux, Nano Lett. 11, 3117 (2011)

${ }^{26}$ J. Anaya, A. Torres, A. Martín-Martín, J. Souto, J. Jiménez, A. Rodríguez, and T. Rodríguez, Appl. Phys. A 113, 167 (2013).

${ }^{27}$ J. Anaya, A. Torres, A. C. Prieto, V. Hortelano, J. Jiménez, A. Rodríguez, and T. Rodríguez, Appl. Phys. A 114, 1321 (2014).

${ }^{28}$ Q. Xiong, G. Chen, G. H. R. Gutierrez, and P. C. Eklund, Appl. Phys. A 85, 299 (2006).

${ }^{29}$ G. S. Doerk, C. Carraro, and R. Maboudian, Raman Spectroscopy for Nanomaterials Characterization (Springer Verlag, Berlin, Germany, 2012), pp. 477-506.

${ }^{30}$ L. Cao, B. Nabet, and J. E. Spanier, Phys. Rev. Lett. 96, 157402 (2006).

${ }^{31}$ A. Torres, A. Martín-Martín, O. Martínez, A. C. Prieto, V. Hortelano, J. Jiménez, A. Rodríguez, J. Sangrador, and T. Rodríguez, Appl. Phys. Lett. 96, 011904 (2010).

${ }^{32}$ R. Singh, E. J. Dailey, J. Ducker, and J. Menéndez, J. Appl. Phys. 110, 124305 (2011).

${ }^{33}$ Z. Zhang, D. C. Dillen, E. Tutuc, and E. T. Yu, Nano Lett. 15, 4303 (2015).

${ }^{34}$ J. Anaya, A. Torres, J. Jiménez, A. Rodríguez, T. Rodríguez, and C. Ballesteros, Symposium L - Photonic and Plasmonic Materials for Enhanced Optoelectronic Performance (Mater. Res. Soc. Symp. Proc., 2014), Vol. 1659, SS11-03.

${ }^{35}$ M. I. Alonso and K. Winer, Phys. Rev. B 39, 10056 (1989).

${ }^{36}$ J. C. Tsang, P. M. Mooney, F. Dacol, and J. O. Chu, J. Appl. Phys. 75, 8098 (1994).

${ }^{37}$ I. H. Campbell and P. M. Fauchet, Solid State Commun 58, 739 (1986).

${ }^{38}$ S. Piscanec, M. Cantoro, A. C. Ferrari, J. A. Zapien, Y. Lifshitz, S. T. Lee, S. Hofmann, and J. Robertson, Phys. Rev. B 68, 241312(R) (2003).

${ }^{39}$ J. L. Pura, J. Anaya, J. Souto, C. Prieto, A. Rodríguez, T. Rodríguez, and J. Jiménez, Nanotechnology 27, 455709 (2016).

${ }^{40}$ A. Potié, T. Baron, L. Latu-Romain, G. Rosaz, B. Salem, L. Montès, P. Gentile, J. Kreisel, and H. Roussel, J. Appl. Phys. 110, 024311 (2011).

${ }^{41}$ A. Picco, E. Bonera, E. Grilli, M. Guzzi, M. Giarola, G. Mariotto, D. Chrastina, and G. Isella, Phys. Rev. B 82, 115317 (2010).

${ }^{42}$ X. Wang, L. Tsybeskov, T. I. Kamins, X. Wu, and D. J. Lockwood, J. Appl. Phys. 118, 234301 (2015).

${ }^{43}$ Sopra database S A, France. 